\title{
ON RESIDUALLY FINITE KNOT GROUPS
}

\author{
BY
}

E. J. MAYLAND, JR.(1)

\begin{abstract}
The residual finiteness of the class of groups of fibred knots, or those knot groups with finitely generated and, therefore, free commutator subgroups, has been known for some time. Using Baumslag's results on absolutely parafree groups, this paper extends the result to twist knots (Whitehead doubles of the trivial knot) and certain other classes of nonfibred knots whose minimal spanning surface has complement with free fundamental group. As a by-product more explicit finite representations, namely cyclic extensions of certain $p$-groups, are obtained for these knot groups and the groups of fibred knots. Finally composites of two such knots also have residually finite groups.
\end{abstract}

0. Introduction. A group is residually finite if the intersection of its normal subgroups of finite index is the identity. The residual finiteness of the groups of fibred knots, or those knot groups with finitely generated and, therefore, free commutator subgroups, has been known for some time [13, p. 63]. We include in $\$ 2$ a modification of the proof of this theorem which gives more explicit information on the structure of a sufficient set of finite homomorphic images. This result is extended, using Baumslag's theorems on parafree groups ([1] and [2]), to those knot groups whose commutator subgroup is an ascending union of parafree subgroups and, therefore, in particular to those knot groups whose commutator subgroup can be built up from a free group by the careful iterated adjunction of a countable sequence of roots. In $\S 3$ we give a brief account of Neuwirth's analysis of the structure of the commutator subgroup of a knot group, enabling us to give in $\$ 4$ some examples of such residually finite knot groups including the groups of the so-called twist knots, or Whitehead doubles of the trivial knot. We also show that any composite of such knots has a residually finite group. Finally, we give a brief indication of how this work might be continued.

We remark that $\mathrm{P}$. Stebe has shown that hose knots have residually finite groups by showing their groups are $\Pi_{c}$ [16], that is for each two elements $g_{1}$ and $g_{2}$ in the group, either $g_{1}=g_{2}^{t}$ or else there is a normal subgroup $N$ of finite index such that $g_{1} \not \equiv g_{2}^{n} \bmod N$ for each integer $n$.

Presented to the Society, January 18, 1972; received by the editors May 25, 1971. AMS 1970 subject classifications. Primary 55A25, 20E25, 55A05; Secondary 20E10.

( ${ }^{1}$ Acknowledgements. This work is essentially the author's doctoral thesis at the University of Wisconsin and I gratefully acknowledge the guidance, assistance, and encouragement of my advisor, Professor Joseph Martin, in its preparation. I would also like to thank Professor Richard Bruck for many helpful conversations.

Copyright () 1972, American Mathematical Society 
We define a cube-with-holes to be the closure of the complement of a regular neighborhood of a finite graph in $S^{3}$. By a theorem of Mal'cev (see [8, p. 415]), a finitely generated residually finite group $G$ is Hopfian, that is every endomorphism from $G$ onto itself is one-to-one. It follows that if $G=\pi_{1}\left(M^{3}\right)$ is also the fundamental group of the cube-with-holes $M^{3}$ and if $f: M^{3} \rightarrow M^{3}$ is a (continuous) mapping such that $f$ restricted to the boundary of $M^{3}$ is the identity, then $f$ induces a monomorphism from $\pi_{1}\left(M^{3}\right)$ to itself, and hence by a theorem of Waldhausen [17] $f$ is homotopic, relative to the boundary of $M^{3}$, to a homeomorphism. (For related material see [3] and [9].)

1. Definitions and notation. We will make use of the following group-theoretical definitions and notations. A presentation for the group generated by $A_{1}, \ldots, A_{n}$ and related by $R_{1}\left(A_{i}\right), \ldots, R_{m}\left(A_{i}\right)$ will be denoted $\left\langle A_{1}, \ldots, A_{n} ; R_{1}\left(A_{i}\right), \ldots, R_{m}\left(A_{i}\right)\right\rangle$. If the groups $H_{1}, \ldots, H_{n}$ and/or elements $B_{1}, \ldots, B_{m}$ are all contained in a supergroup $G$, then by $\mathrm{gp}\left\langle H_{1}, \ldots, H_{n}, B_{1}, \ldots, B_{m}\right\rangle$ we mean the subgroup generated by the $H_{i}$ and $B_{j}$.

The free product $A * B$ of the groups $A=\left\langle a_{1}, \ldots, a_{n} ; R_{1}\left(a_{v}\right), \ldots, R_{p}\left(a_{v}\right)\right\rangle$ and $B=\left\langle b_{1}, \ldots, b_{m} ; S_{1}\left(b_{\mu}\right), \ldots, S_{q}\left(b_{\mu}\right)\right\rangle$ is the group

$$
A * B=\left\langle a_{1}, \ldots, a_{n}, b_{1}, \ldots, b_{m} ; R_{1}\left(a_{v}\right), \ldots, R_{p}\left(a_{v}\right), S_{1}\left(b_{\mu}\right), \ldots, S_{q}\left(b_{\mu}\right)\right\rangle .
$$

If in addition $H=\operatorname{gp}\left\langle U_{1}\left(a_{v}\right), \ldots, U_{s}\left(a_{v}\right)\right\rangle$ is a subgroup of $A$ and

$$
K=\mathrm{gp}\left\langle V_{1}\left(b_{\mu}\right), \ldots, V_{s}\left(b_{\mu}\right)\right\rangle
$$

is a subgroup of $B$ such that $H$ and $K$ are of the same isomorphism type $J$, and the mapping $U_{i}\left(a_{v}\right) \rightarrow V_{i}\left(b_{\mu}\right)$ induces an isomorphism $\phi$ from $H$ to $K$, then

$$
\begin{aligned}
G=\left\langle a_{1}, \ldots, a_{n}, b_{1}, \ldots, b_{m}\right. & \\
& \left.R_{1}\left(a_{v}\right), \ldots, S_{1}\left(b_{\mu}\right), \ldots, U_{1}\left(a_{v}\right)\left[V_{1}\left(b_{\mu}\right)\right]^{-1}, \ldots, U_{s}\left(a_{v}\right)\left[V_{s}\left(b_{\mu}\right)\right]^{-1}\right\rangle
\end{aligned}
$$

is called the generalized free product of $A$ and $B$ with single amalgamated subgroup (under $\phi$ ), or the Schreier product of $A$ and $B$ amalgamating $J$. If $\phi_{1}: J \rightarrow H$ and $\phi_{2}: J \rightarrow K$ isomorphically, then we will abbreviate this notation by

$$
G=\left\langle A * B ; \phi_{1}(J)=\phi_{2}(J)\right\rangle .
$$

For a discussion of generalized free products, see [8], [10], or [11].

If $G$ is a group, $m$ an integer, $g$ an element of $G$ and $\langle x\rangle$ the cyclic group generated by $x$, then we say the group $\left\langle G *\langle x\rangle ; x^{m}=g\right\rangle$ is obtained from $G$ by adjoining an $m$ th root of $g$.

Let $C$ be a nonempty class of groups. We say a group $G$ is residually $C$ if for each nonidentity element $g$ contained in $G$ there exists a group $H$ in $C$ and an onto homomorphism $\tau: G \rightarrow H$ such that $1 \neq \tau(g)$. Equivalently $G$ is residually $C$ if the intersection of all those normal subgroups $N$ for which $G / N$ belongs to $C$ is the unit element of $G$. If the groups of the class $C$ are characterized by a particular property $\pi$, we shall also say that $G$ is residually $\pi$. For example, we shall be con- 
cerned with groups that are residually finite, residually nilpotent, and residually of order a power of a fixed prime $p$.

We will denote the commutator $A^{-1} B^{-1} A B$ of elements $A$ and $B$ by $[A, B]$. For a group $G$, the terms $\gamma_{j} G$ of the lower central series of $G$ are defined by $\gamma_{j} G=\operatorname{gp}\left\langle\left[g_{1}, g_{2}, \ldots, g_{j}\right]\right| g_{i}$ in $\left.G\right\rangle$, where $\left[\cdots\left[\left[g_{1}, g_{2}\right], g_{3}\right] \cdots g_{j}\right]=\left[g_{1}, \ldots, g_{j}\right]$. We may also denote $\gamma_{2} G$ by $G^{\prime}$. The lower central sequence of $G$ is the sequence of quotients $G / \gamma_{2} G, G / \gamma_{3} G, \ldots$ Also $G$ is free nilpotent if $G \cong F / \gamma_{j} F$ for some free group $F$. $G$ is absolutely parafree or parafree (in the variety of all groups) if $G$ has the same lower central sequence as a free group and $G$ is residually nilpotent (see [1] and [2]).

Finally for any group $G$, the subgroup $G^{p^{t}}$ is defined by $G^{p^{t}}=\operatorname{gp}\left\langle g^{p^{t}}\right| g$ in $\left.G\right\rangle$ and, for a given prime $p$, the subgroup $G(i, j)$ by $G(i, j)=\gamma_{j} G \cdot G^{p^{i}}$.

\section{The residual finiteness of certain classes of knot groups.}

TheOREm 2.1 (NeUWIRTH [13, p. 63]). Fibred knots have residually finite groups.

Proof. Let $1 \neq w$ be an element of the group $G$ of a fibred knot $K, Y=\gamma_{2} G$ be the commutator subgroup of $G$, and $t$ be an element of $G$ whose image generates the homology quotient group $G / Y$.

If $w$ does not belong to $\gamma_{2} G$, then $w$ is represented nontrivially in the residually finite (cyclic) group $G / \gamma_{2} G$, implying there is a normal subgroup of finite index in $G$ not containing $w$.

Otherwise $w$ belongs to $Y=\gamma_{2} G$ which is free of rank twice the genus of $K$ and therefore residually nilpotent. It follows that $w$ does not belong to some term $\gamma_{j} Y$ of the lower central series of $Y$, and therefore has nontrivial image in $Y / \gamma_{j} Y$. If, for a given prime $p, Y^{p^{i}}=\mathrm{gp}\left\langle y^{p^{i}}\right| y$ in $\left.Y\right\rangle$, then, by a theorem of Gruenberg [5], the intersection of the $Y^{p^{i}}$ for $i=1,2, \ldots$ is contained in $\gamma_{j} Y$. Hence $w$ is not contained in $Y(i, j)=\gamma_{j} Y \cdot Y^{p^{i}}$ for some $i$. But $Y(i, j)$ is of finite index in $Y$ by a theorem of Mal'cev [7, Vol. II, p. 248]. See Figure 2.1.

Now $Y(i, j)$ is verbal, hence fully invariant in $Y$ and normal in $G$. Furthermore, $t$ acts on $Y$ by conjugation and thereby induces an automorphism on $Y / Y(i, j)$. Clearly, this automorphism has finite order, so we define $t^{\alpha}$ to be the least power of $t$ which centralizes $Y / Y(i, j)$.

In the group $G / Y(i, j)$ the coset $t^{\alpha} \cdot Y(i, j)$ belongs to the center since $\left[t, t^{\alpha}\right]=1$ and $t$ was chosen to commute with $Y / Y(i, j)$. Hence certainly $t^{\alpha} \cdot Y(i, j)$ generates a normal subgroup of $G / Y(i, j)$ and, taking inverse images under the canonical homomorphism, $\operatorname{gp}\left\langle t^{\alpha}, Y(i, j)\right\rangle$ is a normal subgroup of $G$. But $\left\langle t^{\alpha}, Y(i, j)\right\rangle$ is of finite index $\alpha \cdot|Y: Y(i, j)|$ and does not contain $w$. Therefore, $G$ is residually finite, completing the proof of the theorem.

We remark that $Y / Y(i, j)$ is a Burnside group, that is, a free group of rank $2 g$ in the variety of all groups of nilpotence class at most $j-1$ with exponent $p^{i}$. We have thus shown that any element of $Y$ can be represented, considered as an element of $G$, nontrivially in cyclic extensions of "free $p$-groups". We will extend the 


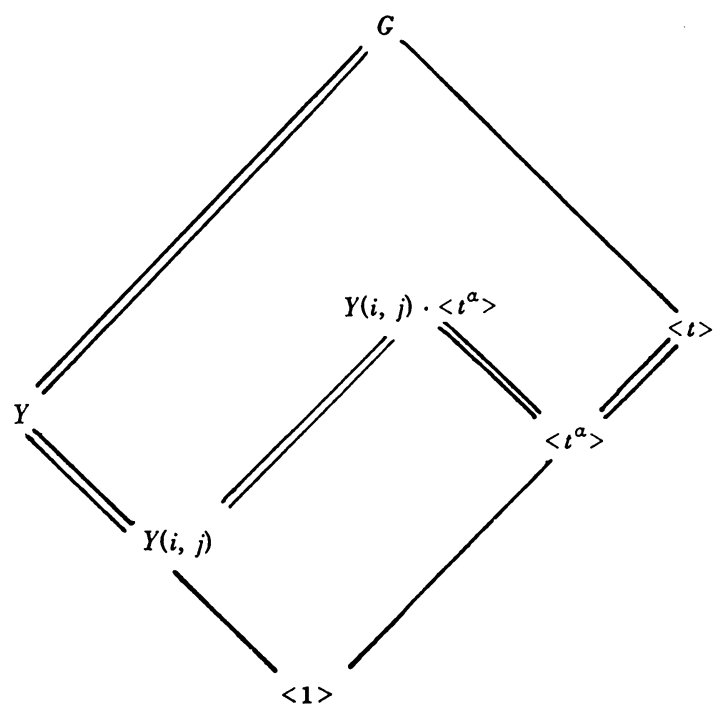

FIGURE 2.1

class of knot groups whose nonidentity elements can be represented in groups ismorphic to the groups $G / Y(i, j)$ of the previous theorem through the use of some results of Gilbert Baumslag. The first result is part of the content of Proposition 2.1, p. 514 in [2].

Proposition 2.2 (Baumslag). Let $A_{1}<A_{2}<\cdots<A_{n}<\cdots<A=\bigcup_{n=1}^{\infty} A_{n}$ be an ascending series of absolutely parafree groups of the same finite rank $r$. If $m_{i}=\left|A_{i+1}\right| A_{i} \cdot \gamma_{2} A_{i+1} \mid<\infty$ for each $i=1,2, \ldots$, then $A$ is residually a finite $p$-group for each prime $p$ dividing at most finitely many $m_{i}$. In particular, for such $p, A / A(i, j)$ $\cong F_{r} / F_{r}(i, j)$ for a free group $F_{r}$ of rank $r$ and the intersection of all the $A(i, j)$ is the identity.

Corollary 2.3. Let $G$ be a knot group and $Y=\gamma_{2} G$. If $Y_{1}<Y_{2}<\cdots<Y_{n}$ $<\cdots<Y=\bigcup_{n=1}^{\infty} Y_{n}$ is an ascending series of absolutely parafree subgroups of $Y$ of the same finite rank such that $m_{i}=\left|Y_{i+1}\right| Y_{i} \cdot \gamma_{2} Y_{i+1} \mid<\infty$, for $i=1,2, \ldots$, and if there exists a prime $p$ dividing at most finitely many $m_{i}$, then $G$ is residually finite.

THEOREM 2.4 (BAUMSLAG, [1, p. 313]). Let $H$ be finitely generated and parafree of rank $r$, let $\langle x\rangle$ be the infinite cyclic group on $x$, and let $q$ be a positive integer which is not divisible by a prime $p$. If $h$ generates its own centralizer in $H$ and if $h \equiv g^{q}$ modulo $\gamma_{2} H$, where $g$ is not a proper power modulo $\gamma_{2} H$, then

$$
G=\left\langle H *\langle x\rangle ; h=x^{p}\right\rangle
$$

is parafree of rank $r$.

COROllary 2.5. Let $G$ be a knot group and $Y=\gamma_{2} G$. If $Y_{1}<Y_{2}<\cdots<Y_{n}$ $<\cdots<Y=\bigcup_{n=1}^{\infty} Y_{n}$ is an ascending series of subgroups of $Y$ such that $Y_{1}$ is 
absolutely parafree of finite rank and each $Y_{n}$, for $n=2,3, \ldots$, comes from $Y_{n-1}$ by adjoining a p $p_{n}$ th root of some element $h_{n-1}$ in $Y_{n-1}$ (where $h_{n-1} \equiv\left(g_{n-1}\right)^{k_{n-1}}$ modulo $\gamma_{2} Y_{n-1} ;\left(p_{n}, k_{n-1}\right)=1$; and $g_{n-1}$ is not a proper power modulo $\left.\gamma_{2} Y_{n-1}\right)$. Then if there exists a prime $p$ which divides at most finitely many of the primes $p_{n}, G$ is residually finite.

Proof. By the previous theorem the $Y_{n}$ are absolutely parafree. Evidently $\left|Y_{n+1} / Y_{n} \cdot \gamma_{2} Y_{n+1}\right|=p_{n}$, so by Corollary $2.3, G$ is residually finite.

3. Constructing the commutator subgroup of a knot group. Given a knot $K$ it is possible to construct a presentation for the commutator subgroup, $\pi_{1}^{\prime}\left(S^{3}-K\right)$, by constructing the corresponding covering space of $S^{3}-K$. (For more details of this analysis, due to Neuwirth, see [13, Chapter IV].) Briefly, let $S$ be an oriented surface of minimal genus spanned by $K$. To form the manifold " $S^{3}$ split along $S$ ", we first extend a triangulation of $S$ to a triangulation of $S^{3}$. Then $M$ is made from a collection of disjoint (closed) 3-simplices which are in one-to-one correspondence with the 3-simplices of our triangulation of $S^{3}$ by identifying two 3-simplices along a (closed) face if the corresponding 3-simplices are incident along the corresponding faces in $S^{3}$, and if this face is not in $S$. $M$ is now a complex containing two copies of $S$ in its boundary, which we arbitrarily order as the "lower" copy ${ }_{1} S$ and the "higher" copy ${ }_{2} S$. The natural covering map $\phi$ from $M$ onto $S$ is performed identically on the interior of $M$ and by identifying the remaining pairs of faces in the boundary of $M$ which correspond to simplices of $S$.

To form the covering space $\left(M^{\infty}, \Phi\right)$, we take countably many copies $M_{i}$ $(i=0,1,-1,2,-2, \ldots)$ of $M$ and join them alternately to the "higher" and "lower" end of a chain, starting at $M_{0}$. In each case the attachment consists of identifying the "higher" copy ${ }_{2} S_{i}$ of $S$ in the boundary of some $M_{i}$ with the "lower" copy ${ }_{1} S_{i+1}$ of $S$ in the boundary of the next higher indexed copy of $M$. We then remove from all stages those 1 -simplices mapped onto $K$ by the associated covering maps $\phi_{i}$. The covering map $\Phi$ is defined as the natural map induced by the restrictions of the $\phi_{i}$ to the complements of the $\phi_{i}^{-1}(K)$. That this covering corresponds to the commutator subgroup follows since exactly those elements of $\pi_{1}\left(S^{3}-K\right)$ which have 0 linking number [15] with $K$ and, therefore, 0 algebraic intersection with $S$ will lift to loops in the covering space.

Let $M^{n}$ denote the $n$-fold "covering" of $S^{3}-K$ obtained at the $n$th stage of this construction (i.e. the $n$-length chain of copies of $M$ with those 1-simplices which correspond to $K$ removed).

In order to follow this construction group-theoretically, we present the fundamental group of $S$, which is free of rank twice the genus of $K$, by

$$
\pi_{1}(S)=\left\langle\xi_{1}, \xi_{2}, \ldots, \xi_{2 g} ;-\right\rangle
$$

and denote the fundamental groups of $M_{i}$ and $M^{n}$ by $X_{i}$ and $X^{n}$, respectively. In $M_{i}$ we let ${ }_{1} h_{i}$. and ${ }_{2} h_{i^{*}}$ denote the inclusion-induced monomorphisms mapping 
respectively the fundamental groups of the lower and higher copies of $S$ in the boundary of $M_{i}$ into $X_{i}$. (See [13, Lemma 4.4.2].)

If we now attach $M_{i}$ and $M_{i+1}$ by identifying the appropriate copy of $S$ in their respective boundaries, then, by the van Kampen theorem, the resulting manifold will have fundamental group a generalized free product of $X_{i}$ and $X_{i+1}$ with amalgamated subgroup $\pi_{1}(S)$. The amalgamating relations set ${ }_{2} h_{i} \cdot(S)$ equal to ${ }_{1} h_{i+1} \cdot(S)$, or they have the form

$$
\begin{gathered}
{ }_{2} h_{i} \cdot\left(\xi_{1}\right)={ }_{1} h_{i+1} \bullet\left(\xi_{1}\right) \\
\vdots \\
{ }_{2} h_{i} \cdot\left(\xi_{2 g}\right)={ }_{1} h_{i+1} \bullet\left(\xi_{2 g}\right) .
\end{gathered}
$$

In particular, there is a natural imbedding of $X_{i}$ and $X_{i+1}$ in the resulting group, as factors of the generalized free product. Similarly each $X^{n}$ is imbedded as a factor in $X^{n+1}$ and the group $\pi_{1}^{\prime}\left(S^{3}-K\right)$ is the direct limit of the $X^{n}$ under these imbeddings. Identifying $X^{n}$ with its image in $\pi_{1}^{\prime}\left(S^{3}-K\right)$ we have

$$
X_{0}=X^{1}<X^{2}<\cdots<X^{n}<\cdots<\pi_{1}^{\prime}\left(S^{3}-K\right) \cong \bigcup_{n=1}^{\infty} X_{n} .
$$

We remark that the groups $X_{i}$ are also imbedded in $\pi_{1}^{\prime}\left(S^{3}-K\right)$, so that $\pi_{1}^{\prime}\left(S^{3}-K\right)$ may be presented by

$$
\begin{aligned}
& \pi_{1}^{\prime}\left(S^{3}-K\right) \cong\left\langle\ldots, X_{-1}, X_{0}, X_{1}, \ldots ;{ }_{2} h_{i^{*}}\left(\xi_{j}\right)={ }_{1} h_{i+1^{*}}\left(\xi_{j}\right)\right\rangle \\
& \quad \text { for } j=1, \ldots, 2 g \text { and } i=0,1,-1,2, \ldots .
\end{aligned}
$$

Then the group $\pi_{1}\left(S^{3}-K\right) / \pi_{1}^{\prime}\left(S^{3}-K\right)$ is isomorphic to the group of covering translations of $M^{\infty}$, which is free cyclic and generated by the shift operator which sends each $M_{i}$ to $M_{i+1}$. If we choose an element $t$ of $\pi_{1}\left(S^{3}-K\right)$ whose image generates this quotient group and so that the action of $t$ on the commutator subgroup induced by conjugation is the same as the action induced by the shift operator (see [13]), then abbreviating the fact that conjugation by $t$ sends $X_{i}$ "identically" onto $X_{i+1}$ by writing $t^{-1} \cdot X_{i} \cdot t=X_{i+1}$, we can present $\pi_{1}\left(S^{3}-K\right)$ as

$$
\begin{array}{r}
\pi_{1}\left(S^{3}-K\right) \cong\left\langle t, \ldots, X_{-1}, X_{0}, X_{1}, \ldots ;{ }_{2} h_{i} \cdot\left(\xi_{j}\right)={ }_{1} h_{i+1} \cdot\left(\xi_{j}\right), t^{-1} \cdot X_{i} \cdot t=X_{i+1}\right\rangle \\
\text { for } j=1, \ldots, 2 g, \text { and } i=0,1,-1,2, \ldots
\end{array}
$$

In the next section we will give some examples of knots for which the groups $X^{n}$ in (3.2) are absolutely parafree but not free, and for which $X^{n+1}$ can be obtained from $X^{n}$ by adjoining finitely many roots which satisfy the hypotheses of Theorem 2.4 .

4. Examples. In this section we will consider only those knots which have a minimal spanning surface whose complement has free fundamental group.

We consider first the so-called twist-knots [18], which may be constructed (see Figure 4.1) from a Whitehead link in a solid torus by cutting the torus open along a meridianal disk, twisting one end through $\rho$ full twists, and re-identifying the 


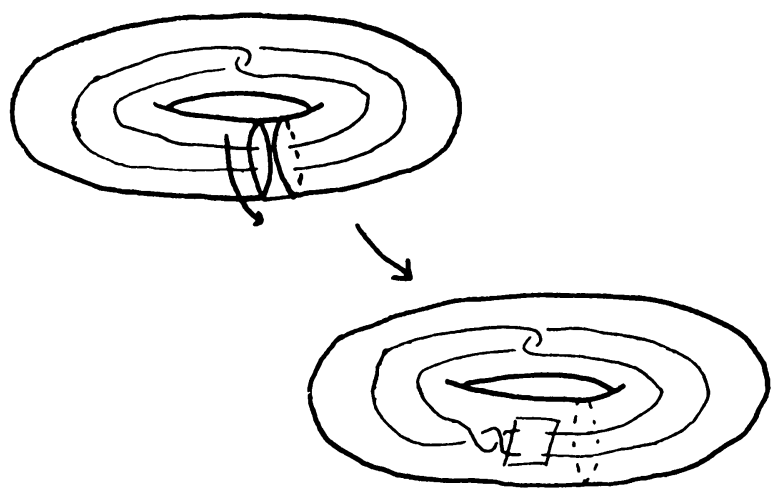

Figure 4.1. Formation of $\rho$-twist knots.

disks. Then the image of the Whitehead link is called a $\rho$-twist knot. By convention $\rho$ is positive if the resulting projection of the knot is alternating.

Of course for $\rho=0$ the resulting knot is trivial, while for $\rho=1$ and $\rho=-1$ we have respectively the four-knot and the trefoil. Another representation of twist knots is given in Figure 4.2. Evidently twist knots bound surfaces of genus one, such

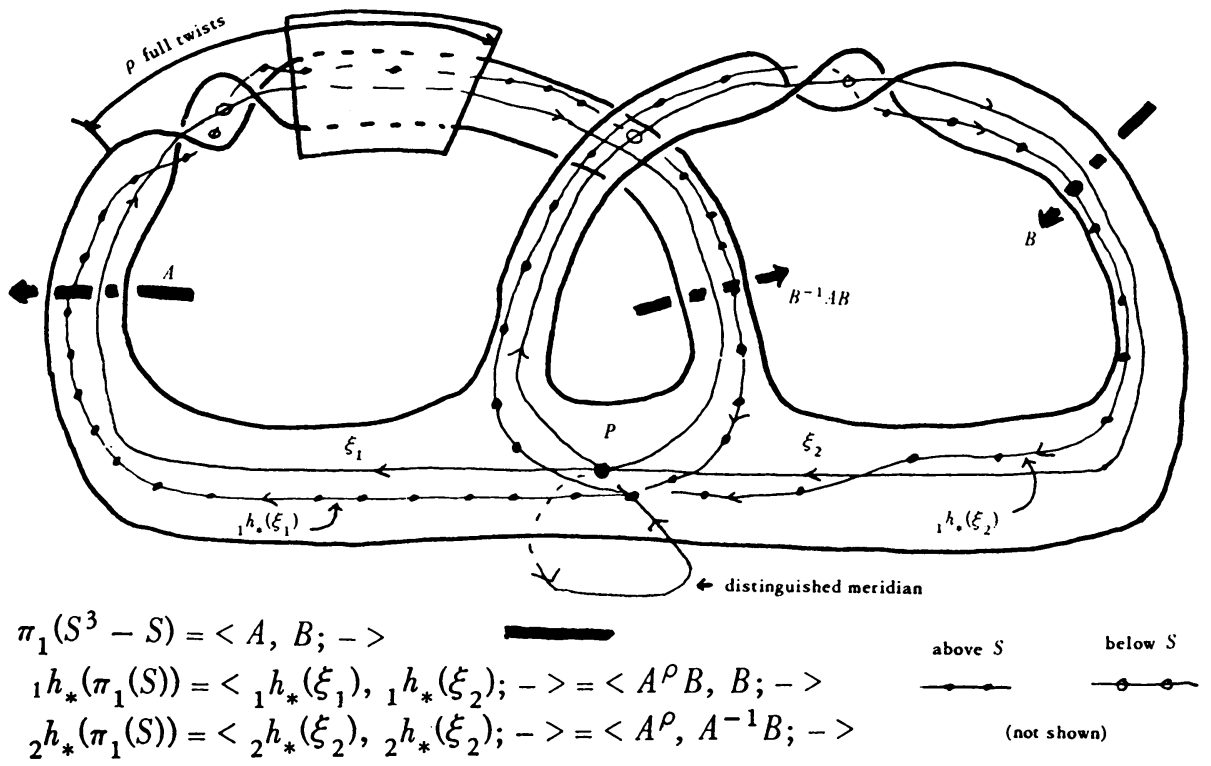

FIGURE 4.2. The surface spanned by a twist knot and associated groups.

that if $\pi_{1}(S)=\left\langle\xi_{1}, \xi_{2} ;-\right\rangle$ and $\pi_{1}\left(S^{3}-S\right)=\langle A, B ;-\rangle$, then

$$
\begin{array}{ll}
{ }_{1} h\left(\xi_{1}\right)=A^{\rho} \cdot B, & { }_{2} h\left(\xi_{1}\right)=A^{\rho}, \\
{ }_{1} h\left(\xi_{2}\right)=B, & { }_{2} h\left(\xi_{2}\right)=A^{-1} \cdot B .
\end{array}
$$


Therefore, the amalgamating relations between $X_{i}$ and $X_{i+1}$ will be (see (3.1))

$$
A_{i}^{\rho}=A_{i+1}^{\rho} \cdot B_{i+1}, \quad A_{i}^{-1} \cdot B_{i}=B_{i+1} .
$$

We remark that if $\rho= \pm 1$, then both ${ }_{1} h_{*}$ and ${ }_{2} h_{*}$ map $\pi_{1}(S)$ onto $\pi_{1}(M)$, or equivalently that the four-knot and the trefoil are both fibred knots [13]. For this reason we will now restrict our attention to twist knots for which $|\rho|>1$.

To simplify the ensuing calculations we will re-index each group $X^{n}$ so that it contains $n$ copies of the group $X$ with consecutive indices $i=1,2, \ldots, n$. Then by (4.1) and $\S 3$ we may present the groups $X^{n}$ for a $\rho$-twist knot by

$$
\begin{aligned}
& X^{2}=\left\langle A_{1}, B_{1}, A_{2}, B_{2} ; A_{1}^{\rho}=A_{2}^{\rho} \cdot B_{2}, A_{1}^{-1} \cdot B_{1}=B_{2}\right\rangle, \\
& X^{n}=\left\langle A_{1}, B_{1}, \ldots, A_{n}, B_{n} ; A_{i}^{\rho}=A_{i+1}^{\rho} \cdot B_{i+1}, A_{i}^{-1} \cdot B_{i}=B_{i+1}\right\rangle \\
& \text { for } i=1,2, \ldots, n-1 .
\end{aligned}
$$

Furthermore, we will delete the extraneous generators $B_{i}$ from the above presentations, using the relations $B_{i}=A_{i}^{-o} \cdot A_{i-1}^{o}$ for $i=2,3, \ldots, n$, and then $B_{1}=A_{2}^{-\rho} \cdot A_{1}^{\rho+1}$. We then have

$$
\begin{aligned}
X^{2} & =\left\langle A_{1}, A_{2} ;-\right\rangle, \\
X^{n} & =\left\langle A_{1}, \ldots, A_{n} ; A_{i}^{\rho}=A_{i-1}^{\rho} \cdot A_{i-2}^{-\rho} \cdot A_{i-1}^{\rho+1}\right\rangle .
\end{aligned}
$$

We are now in a position to prove the main theorem, namely

THEOREM 4.4. Twist knots have residually finite groups.

Proof. We will show first that if the fundamental group $X^{n}$ of the manifold $M^{n}$ occurring in the construction of the covering space corresponding to the commutator subgroup (see §3) is parafree, and if $M^{n+1}$ comes from $M^{n}$ by attaching a higher-indexed copy of $M$ to the "higher" copy of $S$ in the boundary of $M$, then $X^{n+1}$ is parafree. Furthermore $\left|X^{n+1}: X^{n} \cdot \gamma_{2} X^{n+1}\right|=\rho$.

We require the fact that $A_{n}^{\rho} \cdot A_{n-1}^{-\rho} \cdot A_{n}^{\rho+1}$ is primitive in the group $X^{n} / \gamma_{2} X^{n}$. To see this we label the relators by

$$
R(n): A_{n}^{-\rho} \cdot A_{n-1}^{\rho} \cdot A_{n-2}^{-\rho} \cdot A_{n-1}^{\rho+1}
$$

and observe that

$$
R(n) \equiv\left(A_{n}^{-1} \cdot A_{n-1}^{2}\right)^{\rho} \cdot A_{n-1} \cdot A_{n-2}^{-\rho} \quad \bmod X^{n} .
$$

We now define $N$ to be the normal closure of the group generated by $A_{n}^{\rho} \cdot A_{n-1}^{-\rho} \cdot A_{n}^{\rho+1}$ and $\gamma_{2} X^{n}$ in $X^{n}$. It suffices to show that the resulting quotient group is free cyclic.

If, in the original relation $A_{n}^{2 \rho+1} \cdot A_{n-1}^{-\rho} \equiv 1 \bmod N$, we define $t_{1}=A_{n}^{-2} \cdot A_{n-1}$ and substitute $A_{n-1}=A_{n}^{2} \cdot t_{1}$, we obtain

$$
A_{n} \cdot t_{1}^{-\rho} \equiv 1 \text { and } A_{n-1} \equiv t_{1}^{2 \rho+1} \quad \bmod N \text {. }
$$

Equivalently, $A_{n}$ and $A_{n-1}$ generate a cyclic subgroup of $X^{n} / N$. Now by (4.5) and (4.6) we have (for some integer $\left.\kappa_{1}\right) R(n) \equiv t_{1}^{\kappa_{1} \cdot \rho+1} \cdot A_{n-2}^{-\rho} \bmod N$. As before, 
$\left(\kappa_{1} \cdot \rho+1, \rho\right)=1$, so that $t_{1}$ and $A_{n-2}$ (and hence also $A_{n}, A_{n-1}$, and $A_{n-2}$ ) generate a cyclic subgroup of $X^{n} / N$. We continue this process, eventually concluding that gp $\left\langle A_{n}, \ldots, A_{1}\right\rangle$ is cyclic modulo $N$, and therefore, that $A_{n}^{\rho} \cdot A_{n-1}^{-\rho} \cdot A_{n}^{\rho+1}$ is primitive in $X^{n} / \gamma_{2} X^{n}$.

Now let $\rho=p_{1} \cdot p_{2} \cdot \cdots \cdot p_{\nu}$ be a prime factorization of $\rho$. We form the sequence of groups

$$
X^{n}=G_{0}<G_{1}<\cdots<G_{v}
$$

by adjoining to $G_{i}$ (for $i=0,1, \ldots, \nu-1$ ) a root $r_{i+1}$ of order $p_{i+1}$ of the element $g_{i}$ in $G_{i}$. We will have $g_{0}=A_{n}^{\rho} \cdot A_{n-1}^{-\rho} \cdot A_{n}^{\rho+1}$, and $g_{i}=r_{i}$ for $i=1,2, \ldots, \nu-1$.

We have already shown that $g_{0}=A_{n}^{\rho} \cdot A_{n-1}^{-\rho} \cdot A_{n}^{\rho+1}$ is primitive in $G_{0} / \gamma_{2} G_{0}$. Therefore, $g_{0}$ is not congruent to a proper power modulo $\gamma_{2} G_{0}$. It follows, since by a theorem of G. Baumslag [2] any two-generator subgroup of an absolutely parafree group is free, that $g_{0}$ generates its own centralizer in $G_{0}$. Hence by Theorem 2.4, $G_{1}$ is parafree.

$$
G_{1}=\left\langle X^{n} *\left\langle r_{1}\right\rangle ; A_{n}^{\rho} \cdot A_{n-1}^{-\rho} \cdot A_{n}^{\rho+1}=r_{1}^{p}\right\rangle .
$$

Certainly $r_{1}$ is primitive in $G_{1} / \gamma_{2} G_{1}$, so $G_{2}$ is parafree by the same argument, with presentation (deleting $r_{1}=r_{2}^{p_{2}}$ )

$$
G_{2}=\left\langle X^{n} *\left\langle r_{2}\right\rangle ; A_{n}^{\rho} \cdot A_{n-1}^{-o} \cdot A_{n}^{\rho+1}=r_{2}^{p_{1}} \cdot p_{2}\right\rangle .
$$

Continuing in this manner we conclude that $G_{v}$ is parafree with presentation (deleting $r_{v-1}=r_{v}^{p}$ and then substituting $A_{n+1}$ for $r_{v}$ )

$$
G_{v}=\left\langle X^{n} *\left\langle A_{n+1}\right\rangle ; A_{n}^{\rho} \cdot A_{n-1}^{-\rho} \cdot A_{n}^{\rho+1}=A_{n+1}^{\left.p_{1} \cdot p_{2} \cdots \cdot p_{v}\right\rangle}\right. \text {. }
$$

Thus $X^{n+1}=G_{v}$ is parafree. Also $\left|X^{n+1} / X^{n} \cdot \gamma_{2} X^{n+1}\right|=\rho$.

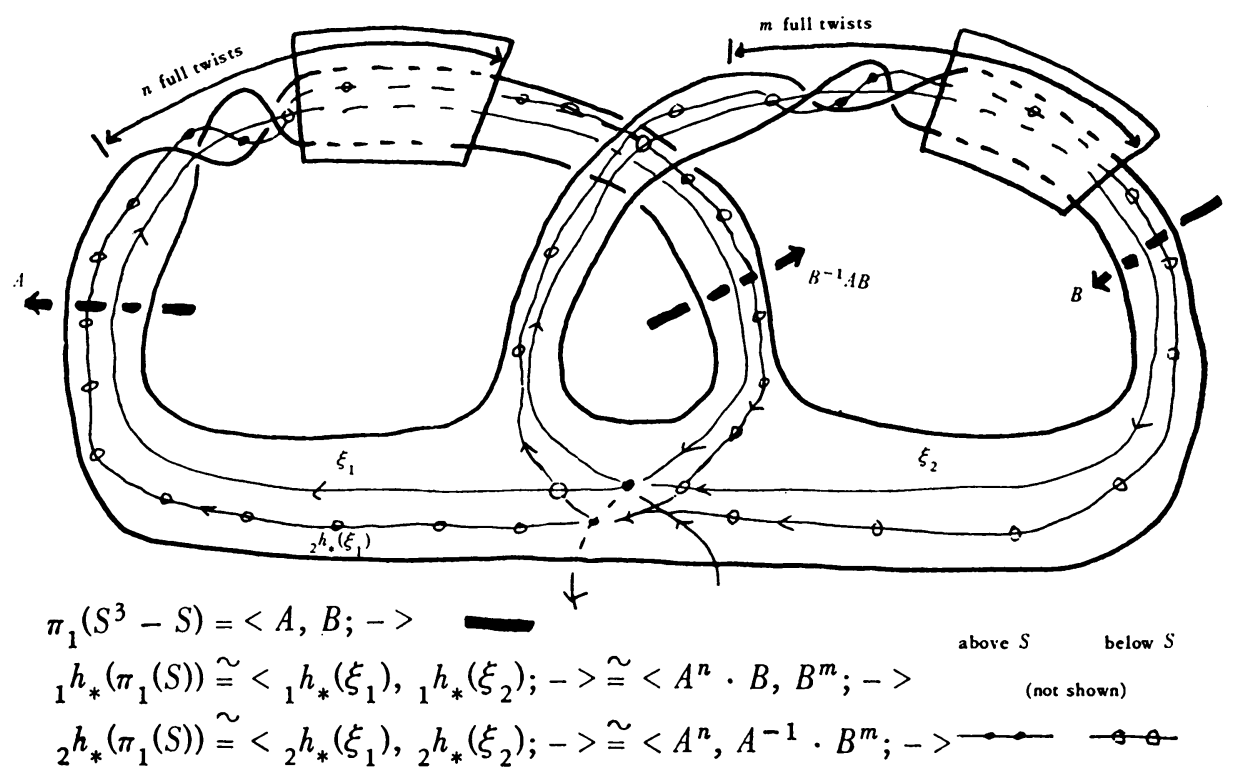

Figure 4.3. The surface spanned by the $\operatorname{knot} K(n, m)$ and associated groups. 
The case that $X^{n}$ is parafree and $M^{n+1}$ comes from $M^{n}$ by attaching a lowerindexed copy of $M$ to the "lower" copy of $S$ in the boundary of $M^{n}$ is similar. Since $X^{1}$ and $X^{2}$ are absolutely free (see (4.3)) we have by induction that each $X^{n}$ in the representation (3.2) for $\pi_{1}^{\prime}\left(S^{3}-K\right)$ as a direct limit of subgroups is parafree. Thus $\pi_{1}\left(S^{3}-K\right)$ is residually finite by Corollary 2.3 . This completes the proof of Theorem 4.4.

Suppose that the class of knots $K(n, m)$ (for integers $n$ and $m$, with $n \cdot m \neq 0$ ) is defined by Figure 4.3. Here

$$
\begin{array}{ll}
{ }_{1} h_{*}\left(\xi_{1}\right)=A^{n} \cdot B, & { }_{2} h_{*}\left(\xi_{1}\right)=A^{n}, \\
{ }_{1} h_{*}\left(\xi_{2}\right)=B^{m}, & { }_{2} h_{*}\left(\xi_{2}\right)=A^{-1} \cdot B^{m},
\end{array}
$$

so the amalgamating relations between $X_{i}$ and $X_{i+1}$ will be

$$
A_{i}^{n}=A_{i+1}^{n} \cdot B_{i+1}, \quad A_{i}^{-1} \cdot B_{i}^{m}=B_{i+1}^{m} .
$$

Under this notation a twist knot with $n$ twists is a $K(n, 1)$ knot. As a corollary of the proof of Theorem 4.4 we present

COROLlARY 4.7. The knots $K(n, m)$ have residually finite groups.

Proof. Following the proof of Theorem 4.4 we show that $A_{u}^{-1} \cdot B_{u}^{m}$ is primitive in $X^{u} / \gamma_{2} X^{u}$ by dividing out the group it generates and showing that the resulting presentation collapses to a free cyclic group. Similarly, we define $Y^{u}=\left\langle X^{u} *\left\langle B_{u+1}\right\rangle\right.$; $A_{u}^{-1} \cdot B_{u}^{m}=B_{u+1}^{m}>$ and show that $A_{u}^{n} \cdot B_{u+1}^{-1}$ is primitive in $Y^{u} / \gamma_{2} Y^{u}$. It then follows that the groups $X^{u}$ and $Y^{u}$ are parafree by induction on $u$ and that the knots $K(n, m)$ have residually finite groups.

For a last example we define a knot $K$ to be the composite or product of two knots $K_{1}$ and $K_{2}$ (written $K=K_{1} \# K_{2}$ ) if there is a 2-sphere $T$ and an $\operatorname{arc} \beta$ contained in $T$ such that (i) $T \cap K=\{x, y\}$ for points $x \neq y$, (ii) $\beta$ is an arc from $x$ to $y$, (iii) ((Int $T) \cap K) \cup \beta$ is a knot of the same type as $K_{1}$, and (iv) ((Ext $\left.\left.T\right) \cap K\right) \cup \beta$ is a knot of the same type as $K_{2}$. By a theorem of Schubert [14] it follows that the minimal surface $S(K)$ for $K$ can be composed from the minimal surfaces $S\left(K_{1}\right)$ and $S\left(K_{2}\right)$ by identifying an arc in the boundary of each. By the van Kampen theorem $\pi_{1}(S(K))=\pi_{1}\left(S\left(K_{1}\right)\right) * \pi_{1}\left(S\left(K_{2}\right)\right)$. Similarly $X(K)=X\left(K_{1}\right) * X\left(K_{2}\right)$. Suppose now that $K_{1}$ and $K_{2}$ both have commutator subgroups, which are unions of their respective parafree subgroups $X^{n}\left(K_{i}\right)$, such that $X^{n+1}\left(K_{i}\right)$ can be obtained from $X^{n}\left(K_{i}\right)$ by the adjunction of finitely many roots. Then starting with the assumption that $X^{n}(K)=X^{n}\left(K_{1}\right) * X^{n}\left(K_{2}\right)$ is parafree, we could adjoin roots to elements involving only generators for $X^{n}\left(K_{1}\right)$ to obtain that $X^{n+1}\left(K_{1}\right) * X^{n}\left(K_{2}\right)$ is parafree, and then adjoin roots of elements involving only generators for $X^{n}\left(K_{2}\right)$ to obtain that $X^{n+1}\left(K_{1}\right) * X^{n+1}\left(K_{2}\right)$ is parafree. Following the proof of Theorem 4.4 we could conclude that $K_{1} \# K_{2}$ has a residually finite group. We have proved 
Proposition 4.8. If $K_{1}$ and $K_{2}$ are knots each of whose commutator subgroup is an ascending union of parafree subgroups $X^{n}$ such that $X^{n+1}$ can be obtained from $X^{n}$ by the adjunction of finitely many roots, then the knot $K_{1} \# K_{2}$ has a residually finite group.

5. Remarks. There are many other knots whose groups can be shown to be residually finite by this method. However, checking that the various elements of which we take roots are actually primitive at each stage is very tedious. The next step in this study would be a lemma stating that if $X^{2}$ can be built up from $X^{1}$ by adjoining roots of primitive elements in $X^{1} / \gamma_{2} X^{1}$, then $X^{n+1}$ can be built up from $X^{n}$ by adjoining roots of elements primitive in $X^{n} / \gamma_{2} X^{n}$.

Many knots $K$ have a minimal spanning surface with complement having a free fundamental group, but having amalgamating relations (3.1) which do not immediately allow the construction of $X^{2}$ from $X^{1}$ by adjoining roots. Perhaps the groups $X^{n}$ for such knots can be shown to be parafree by some other method.

\section{REFERENCES}

1. G. Baumslag, Groups with the same lower central sequence as a relatively free group. I: The groups, Trans. Amer. Math. Soc. 129 (1967), 308-321. MR 36 \#248.

2. - Groups with the same lower central sequence as a relatively free group. II: Properties, Trans. Amer. Math. Soc. 142 (1969), 507-538. MR 39 \#6959.

3. D. B. A. Epstein, The degree of a map, Proc. London Math. Soc. (3) 16 (1966), 369-383. MR 33 \#700.

4. R. H. Fox, A quick trip through knot theory, Topology of 3-Manifolds and Related Topics (Proc. The Univ. of Georgia Inst., 1961), Prentice-Hall, Englewood Cliffs, N.J., 1962, pp. 120-167. MR 25 \#3522.

5. K. W. Gruenberg, Residual properties of infinite soluble groups, Proc. London Math. Soc. (3) 7 (1957), 29-62. MR 19, 386.

6. M. Hall, The theory of groups, Macmillan, New York, 1959. MR 21 \#1996.

7. A. G. Kuroš, Theory of groups, GITTL, Moscow, 1953; English transl., Vols. I, II, Chelsea, New York, 1956. MR 15, 501; MR 18, 188.

8. W. Magnus, A. Karrass and D. Solitar, Combinatorial group theory: Presentations of groups in terms of generators and relations, Pure and Appl. Math., vol. 13, Interscience, New York, 1966. MR 34 \#7617.

9. D. R. McMillan, Jr., Boundary preserving mappings of 3-manifolds, Topology of Manifolds, Markham, Chicago, Ill., 1969.

10. B. H. Neumann, An essay on free products of groups with amalgamations, Philos. Trans. Roy. Soc. London Ser. A 246 (1954), 503-554. MR 16, 10.

11. H. Neumann, Generalized free products with amalgamated subgroups. I. Definitions and general properties, Amer. J. Math. 70 (1948), 590-625. MR 10, 233.

12. - Generalized free products with amalgamated subgroups. II. Subgroups of generalized free products, Amer. J. Math. 71 (1949), 491-540. MR 11, 8.

13. L. Neuwirth, Knot theory, Princeton Univ. Press, Princeton, N.J., 1965.

14. H. Schubert, Die eindeutige Zerlegbarkeit eines Knotens in Primknoten, S.-B. Heidelberger Akad. Wiss. Math.-Nat. Kl. 1949, no. 3, 57-104. MR 11, 196. 
15. H. Seifert and W. Threlfall, Lehrbuch der Topologie, Chelsea, New York, 1947.

16. P. Stebe, The residual finiteness of a class of knot groups, Comm. Pure Appl. Math. 21 (1968), 563-583.

17. F. Waldhausen, On irreducible 3-manifolds which are sufficiently large, Ann. of Math. (2) 87 (1968), 56-88. MR 36 \#7146.

18. J. H. C. Whitehead, On doubled knots, J. London Math. Soc. 12 (1937), 63-71.

Department of Mathematics, University of Wisconsin, Madison, Wisconsin 53706

Current address: Department of Mathematics, York University, Toronto 12, Ontario, Canada 